\title{
Filosofía social: Historia, estado actual y potencialidades
}

\author{
Social philosophy: History, current status, and future potential
}

\section{Harold Edmundo Mora Campo}

Profesor de Tiempo Completo adscrito al programa de Derecho de la UCEVA, catedrático de la Universidad del Valle sede Buga y Tuluá, Abogado de la Universidad Santiago de Cali, Candidato a Doctor en Filosofía de la Universidad del Valle, Magíster en Educación de la Universidad San Buenaventura sede Cali, Magíster en Filosofía del Derecho Contemporáneo de la Universidad Autónoma de Occidente de Cali, en convenio con la Universidad Carlos III de Madrid, Especialista en Derecho Procesal de la Universidad Pontificia Bolivariana de Medellín, en convenio con la Universidad San Buenaventura Cali.

Email: haroldmoracampo@gmail.com

Doi: https://doi.org/10.22267/rceilat.204647.92

\begin{abstract}
Resumen
La filosofía social tiene potencialidades independientemente de la filosofía normativa, por cuanto podrá justificar en el futuro, la pretensión de una antropología débil y formal, a partir de la teoría del reconocimiento, dado que en la actualidad la filosofía social cuenta con un sustento histórico, que unido a la dimensión antropológica referida han de asegurar su supervivencia en el futuro. Para desarrollar este supuesto, este escrito se divide en tres partes: I) Historia de la filosofía social; la filosofía ha experimentado en los últimos doscientos años, un proceso de diferenciación que ha originado varias subdisciplinas. Por ello, si bien subsiste la tripartición clásica: filosofía teórica, filosofía práctica y filosofía estética, en la práctica han surgido otras divisiones que difícilmente se ajustan al precitado esquema; II) Un análisis de las patologías sociales, que implica que ya no debe ser el Estado, sino la sociedad la que se separa lentamente de éste. Así emerge la filosofía social, como una perspectiva ética, que se ocupa del estudio de las patologías sociales, que provocan luchas sociales que procuran el reconocimiento y la emancipación; III) potencialidades de dicha disciplina hacia el futuro. En esta dirección, aquello que se debe asumir como "normal" sobre una forma de vida social que posibilite la emancipación, se validaría en la medida en que los integrantes de la sociedad misma, logren un consenso sobre el deseo de asumir los desarrollos sociales por medio de una formación de voluntad organizada democráticamente.
\end{abstract}

Palabras clave: Filosofía social; Teoría del reconocimiento; Patologías sociales; Emancipación; Democracia. 


\begin{abstract}
Social philosophy has a future independent of normative philosophy, which could eventually lead to the pretense of a weak and formal anthropology. Based on recognition theory and given that current social philosophy has a historical basis, together with the stated anthropological dimension it will ensure its survival in the future.

This article is divided into three parts: I) History of social philosophy; over the last two hundred years, philosophy has undergone a process of differentiation that has resulted in the creation of several sub-disciplines. For this reason, although the classical tripartition (theoretical philosophy, practical philosophy and aesthetic philosophy) subsists, in practice, other divisions have arisen that hardly fit the aforementioned scheme; II) an analysis of social pathologies, which implies that it should no longer be the State, but Society that slowly separates from it. This is how social philosophy emerges, as an ethical perspective, used to study social pathologies, which provoke social struggles that seek recognition and emancipation; III) potentialities of said discipline for the future. What should be assumed as "normal" about a social way of life that enables emancipation would be validated to the extent that the members of society itself come to a consensus on the desire to assume social development through democratic organization.
\end{abstract}

Keywords: Social philosophy; Recognition theory; Social pathologies; Emancipation; democracy.

\section{Introducción}

En este escrito se sostiene que la filosofía social tiene potencialidades sin tener que generar lazos de dependencia con la filosofía normativa, por cuanto podrá justificar en el futuro, la pretensión de una antropología débil y formal, esencialmente a partir de los estudios de Honneth (2011) con su teoría del reconocimiento, dado que en la actualidad la filosofía social cuenta con un sustento histórico, que unido a la referida dimensión antropológica han de asegurar su supervivencia en los días por venir. Con el fin de desarrollar este supuesto, este escrito se divide en tres partes: I) Un recorri- do histórico de la filosofía social, que ilustra las fases de su evolución, II) Un análisis de las patologías sociales y de la situación actual de la filosofía social, que describe los problemas que ésta ha enfrentado y que se solventarán con las previsiones que se describen en el punto III): potencialidades de dicha disciplina hacia el futuro.

\section{I) Recorrido histórico de la filosofía social}

Honneth sostiene que como todos los campos del conocimiento teórico, la filosofía ha experimentado en los últimos doscientos años, un proceso de diferenciación que ha origina- 
do una diversidad de subdisciplinas y campos específicos de estudio. En tal virtud, si bien todavía subsiste la tripartición clásica: filosofía teórica, filosofía práctica y filosofía estética, en la práctica han surgido otras divisiones que difícilmente se ajustan al precitado esquema. En consecuencia, se dificulta trazar los límites entre la filosofía moral, la filosofía política, la filosofía de la historia, entre otras. Por tanto, dada la indeterminación del nexo de la filosofía social con los campos cercanos de conocimiento, según las necesidades, en algunas ocasiones, su rol se reduce a una pieza normativa, que procura complementar la sociología; en otros escenarios se arroga una función de interpretación, que se asemeja a un diagnóstico contemporáneo (Honneth, 2011, p.2).

\section{De Rousseau a Nietzche: Génesis del planteamiento filosófico social}

Según Honneth, si bien Thomas Hobbes dio el nombre a la disciplina a mediados del siglo XVII, esta en sentido propio solo fue fundada cien años después por Jean-Jacques Rousseau. Hobbes se interesó por las condiciones jurídicas que permitirían al Estado absolutista asegurar la estabilidad y autoridad para la pacificación de la guerra civil religiosa. En su Leviatán, se centra en la mecánica para garantizar en el contexto de conflictos e intereses, la supervivencia del orden estatal. Luego, Rousseau (2005) a mediados del siglo XVIII, cuando escribe su Discurso sobre la desigualdad desecha la preocupación de Hobbes por dicho orden y concentra su interés en las causas que conducirían a la generación del orden determinado por la sociedad burguesa, signado por la presión de la competencia económica y social, que dio paso a prácticas basadas en el engaño, la envidia y demás acciones dirigidas a obtener ventaja personal a expensas del bienestar del otro. Estas prácticas atrajeron su atención para indagar si dicha sociedad en conjunto, ofrecía a los sujetos las condiciones para "vivir una vida buena y exitosa". Con el cambio de perspectiva de Rousseau frente a la de Hobbes, se direcciona el proyecto moderno de una filosofía social, que en oposición a la filosofía política, y con el liderazgo de Rousseau, ya no cuestiona las condiciones de un orden social correcto o equitativo, sino que estudia las restricciones impuestas por la nueva forma de vida a la autorrealización del ser humano (Honneth, 2011, p. 77). No en vano, para Grueso (2018) no es tarea de los filósofos pontificar el mundo ni definir de manera taxativa la justicia o generar teorías sobre la misma.

Retomando la versión de Roussseau, vale resaltar su crítica al incremento de las necesidades que con el transcurrir del tiempo han invadido a los individuos, aumento que parece acompañado de una pérdida de la libertad individual, en contraste con el ideal de un estado prehistórico, en el que el ser humano debió haber vivido en una situación natural de autosuficiencia, probablemente con prevalencia de la anomia (ausencia o incongruencia de las normas sociales), pero con el paso del tiempo y con el crecimiento demográfico, que conlleva 
la ineludible interacción con el otro, surgió el conflicto y con ello, las patologías sociales, que obstaculizan la autorrealización del individuo. Ante este fenómeno, la crítica de Rousseau (2005) no solo se restringe a la injusticia social, sino a toda una forma de vida. Para ratificar lo dicho, en la primera parte de su obra Discurso sobre la desigualdad, con base en información empírica, presenta una imagen del estado de naturaleza de los seres humanos, que le sirve en la segunda sección de dicha obra como un marco interesante en contrastes, derivados de la mencionada evolución, para abordar y resaltar las patologías modernas. Por ello, piensa que el ser humano antes de toda socialización debió haber vivido 'en sí mismo'; con esta frase quiere decir que debido al aislamiento en el estado de naturaleza, ocasionado por los escasos escenarios para interacción, debió haber actuado motivado por situaciones de forma independiente a las expectativas del otro.

Honneth refiere que Rousseau centra su relato del estado de naturaleza en dos cualidades primigenias del ser humano. Conforme a esta versión, el hombre, antes de hacer el tránsito del estado de naturaleza al de socialización, debió haber actuado por el impulso de autoconservación y por la capacidad de compasión. Con la primera capacidad, esto es, el amour de soi (amor de sí), se hace alusión al mínimo de autofijación narcisista, requerido para la supervivencia individual en un medio hostil; con la segunda, la pitié (piedad, compasión) se hace referencia al afecto natural que les permite a los seres humanos y en menor grado a los animales, reaccionar cuando miran que sus congéneres sufren (Honneth, 2011, p.80).

De lo expuesto se deriva que el ser humano no puede restringirse a una dimensión físico biótica, sino que su estructura contempla también un fuerte componente afectivo, que contempla las prenombradas nociones de amor de si y piedad, pero también la ansiedad, el asombro, la preocupación, dimensiones que provocan cambios en el modo de pensar, de sentir, de actuar y de concebir el mundo, mutaciones procedentes del avance de la sociedad y del surgimiento de nuevos fenómenos, como la globalización. A esta estructura, bien se podría agregar la dimensión espiritual, que si bien para algunos resulta irrelevante, en el marco de esta discusión tiene su cifra; en cuanto que el mismo Platón (1966) considera que una de las funciones centrales de la educación radica en la formación general del espíritu, formación que debe ser anterior a cualquier especialización técnica. A juicio de Honneth, las consideraciones antes anotadas permiten afirmar que Rousseau se ha convertido en el fundador de la filosofía social moderna, dado que su propuesta de considerar la forma original de existencia del individuo, en contraste con su situación actual, plena de patologías sociales, minimiza su libertad. De este modo, aportó un criterio de comparación con el desarrollo humano y abrió un espacio para una de las pocas alternativas de empiria que le quedan a la filosofía social (Honneth, 2011, p. 84). 
Para Honneth, Hegel a diferencia de Rousseau no se preocupó por la pérdida de libertad subjetiva, puesto que esta en su opinión, no caracterizaba a la sociedad de la época. Más bien, centró su interés en el "efecto destructivo que parte del proceso de aumento desmesurado del particularismo individual”. Por ello, se interesó por los fenómenos empíricos de la individualización social y por la apatía política. Pese a lo anterior, Hegel comparte con Rousseau, lo referente a los desarrollos históricos deficientes, porque estos suponen un peligro social, en la medida en que restringen las condiciones de una vida buena. A diferencia de Rousseau y de Hegel, Marx considera que el sujeto humano solo alcanza la autorrealización por medio del trabajo. Luego Nietzsche, a juicio de Honneth considera que la vida social de su época fue bastante perturbada, debido a una disposición básica nihilista, derivada de asumir que toda orientación hacia valores optimistas se condiciona por procesos reflexivos, que no son marginales, sino la expresión sintomática de una patología cultural. Con este presupuesto se desarrolla un enfoque que constituye un elemento fundamental en el avance de la filosofía social moderna (Honneth, 2011, p. 85).

\section{Entre antropología y filosofía de la historia. La filosofía social después del surgimiento de la sociología}

Una versión honnetiana revela que la filosofía del siglo XIX estuvo marcada por la influencia de Rousseau, Marx y Nietzsche; sin embargo, el es- tudio de las patologías se traslada de dicha filosofía a la sociología, campo en proceso de formación, sujeto por tanto a diversas denominaciones como la "ciencia de la moral" o de la "cultura"; y conminado a contribuir a la reparación práctica de la crisis ética, mediante la explicación de su génesis. En esta perspectiva la tarea de los primeros sociólogos se puede comparar con las metas propuestas por Hegel en su filosofía, distanciándose de éste por las determinaciones sustanciales que esgrime para caracterizar el peligro amenazante; esto es, el proceso que explota con la crisis ética de la época, que ya no se identifica por un aumento de la atomización y la disociación, sino por un detrimento de orientación moral en general. Después de la unión de la filosofía social con la sociología, entró en juego la obligación de la comprobación empírica, hecho que resaltó el nexo estrecho entre dicha filosofía y el pensamiento filosófico histórico (Honneth, 2011, p. 96).

Posteriormente, Weber, al decir de Honneth con la categoría de "compromiso valorativo" presentó una interpretación de las ciencias, que atribuye la dimensión de valoraciones éticas a sus condiciones trascendentales de cualquier campo temático, al tenor de valores determinados. En este marco la "neutralidad valorativa" implica una invitación a rendir cuentas a la conciencia de los propios compromisos valorativos de uno; no obstante, esto no clarifica la forma de justificar la realidad de su validez. Estas incoherencias sumen a la filosofía social en una crisis metodológica difícil de su- 
perar, tanto es así que surge la pregunta ¿Cómo era dable continuar persiguiendo el objetivo de determinar y debatir desarrollos sociales deficitarios, si ya no se cuenta para ello con criterios de valoración universales?, preocupación que se solventa con los modelos interpretativos de la sociología, que permitieron explicar algunas patologías sociales, como las del capitalismo. Estos modelos sociológicos tuvieron su incumbencia en el marco de la filosofía, en tanto que aseguraron premisas universalistas, en oposición a los desafíos del relativismo ético. En tal virtud, con la antropología filosófica por un lado, se originó una escuela teórica, que incluyendo investigaciones empíricas, procuraba aclarar la pregunta planteada por Rousseau y Humboldt: ¿qué cualidades universales caracterizan al ser humano frente a los animales? Por otro lado, posiblemente debido a la guerra mundial, un nuevo interés emergió por aquella forma de pensamiento filosófico-histórico, que el historicismo consideraba superado. En consecuencia, la dimensión antropológica y la filosófico-histórica, que siempre habían desempeñado un papel cardinal en la filosofía social, recuperan su esencia y en los años veinte del siglo XX, se consolidaron como corrientes teóricas para retomar el terreno de las premisas universalistas (Honneth, 2011, p. 97).

\section{II) Patologías sociales y situación actual de la filosofía social}

Conforme Honneth, para Kant la escisión entre moral y ética ocurre en el ámbito de la filosofía moral. Rous- seau cuestiona esto desde una doble perspectiva: por una parte se formaliza radicalmente la visión aristotélica, en cuanto que ya no se habla más de los fines de autorrealización humana, dados por la naturaleza, sino solo de las condiciones que habilitan esta autorrealización; y por otra parte, ya no debe ser el Estado, sino la sociedad la que se separa lentamente de éste, la que se interroga sobre las condiciones que obstaculizan la realización de la vida humana. De esta manera emerge la filosofía social, como representante de una perspectiva ética en un nuevo terreno de la sociedad que se empieza a constituir, que conlleva entre otras cosas, el estudio de las patologías sociales. Estas a su vez provocan luchas sociales que procuran el reconocimiento, dando forma de este modo a un horizonte emancipatorio, que se va abriendo como resultado de tales luchas. Se podría decir, incluso, que estas indican a la humanidad el rumbo a seguir (Honneth, 2011, p. 113). En este entorno, al filósofo social solo le resta procurar auscultar los conflictos y las patologías que subyacen en ellos, puesto que a dicho filósofo no le compete prescribir lo que deben hacer los seres humanos, comenta Grueso (2018).

Honneth, de igual manera manifiesta que la filosofía social desde sus inicios no se sumerge en pretensiones individuales, sino debido a su planteo metodológico gira en torno a exigencias formales, referentes a la explicación de lo bueno; ya que se trata de encontrar criterios para la normalidad de las relaciones sociales; en esta perspectiva se han de valorar como 
exitosas, ideales o sanas, las formas de organización de lo social, que le sirvan al individuo para una realización no deformada de sí mismo. Esta afirmación, no obstante, genera la pregunta sobre qué se ha de entender por bueno o adecuado, una interrogación por demás tormentosa, sujeta a diversos criterios, que pueden difuminar su objetividad. Para contrarrestar esto, las investigaciones desarrolladas por pensadores como Nietzsche, Foucault, Weber, Arendt y las que sigue adelantando el mismo Honneth, con sustento en la estructura de las relaciones modernas de poder, la aceptación social de las normas, la tendencia de las pretensiones de conocimiento, el desarrollo de la historia, los fenómenos que emergen, debido al avance de la sociedad y de la ciencia y los criterios generales sobre la normalidad de la vida social, la cultura, aspectos en los que subyace toda una compleja normatividad, relativa a lo bueno, lo sano, lo exitoso, etc. han de servir para desarrollar criterios sobre lo adecuado para potenciar la autorrealización exitosa de los individuos (Honneth, 2011, p. 118).

\section{III) Potencialidades de la filosofía so- cial hacia el futuro}

Con base en los estudios de Honneth, dichas potencialidades se pueden sintetizar así:

La primera opción de justificación de juicios éticos, acordes con la filosofía social, se refiere al intento de procedimentalización de la ética. Honneth considera que en los escritos recientes de Habermas se aclaran preguntas éticas, de la misma forma que ocurre con los discursos prácticos, algo que hasta ahora solo era viable para los problemas de justicia. En esta dirección, aquello que se debe asumir como "normal" o "ideal" sobre una forma de vida social, se validaría en la medida en que los integrantes de la sociedad misma, logren un consenso sobre el deseo de asumir los desarrollos sociales por medio de una formación de voluntad organizada democráticamente. A juicio de Londoño 2015 (p.p. 101-131), Habermas con este enfoque pretende solventar el problema de la legitimidad, esto es, que las normas han de regir la vida de los ciudadanos, han de ser producidas por ellos mismos, en lugar de ser impuestas por gobiernos totalitarios. Honneth cree que esta propuesta habermasiana diluiría la filosofía social como disciplina teórica en sí misma, puesto que la competencia interpretativa estaría en manos de los afectados, quienes por si mismos tendrían que decidir como miembros de una sociedad concreta, sobre lo que debe ser considerado como patológico en su vida social. Para obviar esto, según Honneth, el mismo Habermas ha diseñado una estrategia de argumentación, que se debe considerar como una segunda alternativa de justificación de los juicios éticos. De este modo, Habermas en su Teoría de la acción comunicativa establece que la misma teoría de la sociedad debe señalar el umbral crítico con base en el cual la invasión de imperativos sistémicos en el mundo de vida social, debe ser asumida como una patología social. La distinción entre lo normal 
y lo patológico deviene de una antropología débil y formal, en cuanto la prenombrada distinción se subsume en una pragmática universal, que procura demostrar una forma originaria de práctica humana de habla, como prerrequisito de la reproducción social, hecho que reitera la importancia del lenguaje, no solo como medio de comunicación, sino también como un sistema, que por medio de la racionalidad comunicativa interviene en los procesos de ordenamiento social. La prenombrada distinción implica que el proyecto de una antropología de carácter restringido, ha de reconstruir unas pocas condiciones básicas para la vida humana.

En la actualidad, la opción planteada al final del párrafo precedente, sugiere Honneth, se puede concretar con los estudios de Martha Nussbaum y Charles Taylor, cuyas obras dan forma a la tercera opción, esto es, a una filosofía social del mundo contemporáneo; los mencionados estudios, se pueden sumar a las obras de Habermas y Foucault. Conforme a Honneth (2011), Taylor en su libro Sources of the self (Fuentes del yo) toma como base, en cierto sentido, una "justificación históricamente relativizada de la ética”; según este precepto, solo el hecho de recobrar hermenéuticamente el sentido de los valores éticos por los cuales se orienta la modernidad en su autocomprensión cultural, ha de suministrar información sobre los desarrollos sociales que deben ser considerados como patologías sociales. Siguiendo a Honneth, esta asunción concuerda con las consideracio- nes de Foucault, para quien, el criterio con que la filosofía social diagnostica y discute sobre perturbaciones sociales, solo registra una validez históricamente limitada, en la medida en que se puede aplicar a una era histórica, cuyas decisiones éticas previas han de arrogarse de manera evidente.

Por otro lado, la antropología entendida en sentido general como la ciencia que se ocupa del origen del ser humano, de su desarrollo como especie social y de los cambios en su conducta a través del tiempo, lleva a pensar que el ligamento de esta con la historia aportará sus frutos, puesto que esta última ha creado sus propios recursos analíticos y tradiciones disciplinarias, cuya aplicación ha resultado muy fructífera para el conocimiento de múltiples caras del pasado. Con Miguez (2012) se diría que historia y antropología "caminan de la mano", en tanto que la primera siempre ha de recurrir a la extensa producción de la segunda para generar los marcos analíticos y metodológicos, que viabilicen sus análisis de contexto y hagan más comprensibles sus relatos.

No de menor importancia en esta discusión y con profundas implicaciones futuras son los estudios de Honneth sobre el reconocimiento, quien bien se podría decir, lidera en la actualidad el presente y el futuro de la filosofía social. Grueso (2015) manifiesta que Honneth se focaliza en el entendimiento hegeliano de las relaciones humanas, que supone que el reconocimiento surge de manera diferente en entornos también diferentes de la vida 
humana; al interior de los nexos familiares, donde somos reconocidos como seres necesitados de cuidado y afecto; en las relaciones sociales, en las que el reconocimiento se funda en el derecho y donde somos asumidos como personas legales y en el Estado, que en sentido hegeliano, es donde logramos el reconocimiento como individuos concretos universales.

De lo dicho se desprende que para Honneth, el reconocimiento no se puede circunscribir a una dimensión redistributiva de tipo material; puesto que su propuesta pone de relieve que un miembro de la sociedad solo es integrado en ella por medio de mecanismos de reconocimiento mutuo, pero que estos resultan controvertidos $\mathrm{y}$, por ello generan conflictos, que resultan en una lucha por el reconocimiento; con estos argumentos pretende remediar el déficit sociológico de la teoría crítica. De igual modo, para Honneth, el reconocimiento precede al entendimiento comunicativo, esto es, el progreso que experimentan los sujetos hacia un pleno reconocimiento recíproco de sus personalidades y de ellos mismos como ciudadanos y seres dignos, no ocurre sencillamente en los discursos institucionalizados, sino que dicho reconocimiento debe adquirir la forma de costumbres de percepción, de algo inherente al individuo como integrante de la sociedad, de tal forma que en este entorno se debería hablar de racionalizaciones, en tanto que sean capacidades potenciadas que faciliten revelar cualidades humanas de valor, Honneth (2011, p. 180).
Uno se podría atrever a decir que con el nexo entre historia y antropología se evita una visión monológica de la filosofía social, aserción que concuerda con las corrientes actuales del conocimiento, que miran con reserva la hiperespecialización, la segmentación desmedida de los saberes, las verdades definitivas y únicas, entre otros aspectos, puesto que dichas corrientes asumen el conocimiento en evolución permanente, por tanto sujeto a procesos de construcción, de reconstrucción y de resignificación, que en muchos casos hacen impredecible su decurso. En esta perspectiva subyace la pluralidad, la diferencia, la posibilidad de preservar la identidad al interior de una comunidad. Los planteos precedentes sincronizan de igual modo con los estudios de De Bono (1986), quien sostiene que diferentes puntos de vista, debidamente sustentados, potencian una mejor comprensión de un hecho o fenómeno. Al tenor de esta afirmación, cabe la mención que de Young hace Grueso (2008), en cuanto que el ideal de una sociedad justa y la institución en la que se eliminan las diferencias, no es del todo realista; puesto que la justicia en una sociedad segmentada en grupos, de una u otra forma requiere la igualdad social de dichos colectivos y el recíproco reconocimiento y afirmación de las diferencias de tales agrupaciones.

En todo caso, el fin último de estas consideraciones debe ser la facilitación de la autorrealización, tendiente a lograr una vida buena y digna, aspiración que se ha de viabilizar por medio del reconocimiento como lo 
entiende Honneth, es decir, que además de una redistribución material, incluya las caras ocultas que afectan el logro de un reconocimiento total, caras que entre otras incluyen el padecimiento, la discriminación, la humillación. Después de todo como afirma Melich (2001), vivimos en un mundo que no hemos creado y que por tanto no nos pertenece; es decir, somos simples itinerantes del mismo. Por tanto, ante la brevedad de la existencia conviene más bien, dar a cada cual lo suyo y desterrar las referidas caras ocultas, puesto que estas manifestaciones invisibles, pero nocivas y dolorosas, obstaculizan la plena autorrealización del individuo y por ende obstruyen su reconocimiento integral, integralidad que defiende la filosofía social y que por lo dicho, se ha de potenciar de manera ostensible en el futuro.

\section{Conclusión}

La filosofía en los últimos doscientos años ha experimentado un proceso de diferenciación que ha dado origen a una diversidad de subdisciplinas y campos específicos de estudio; debido a esto, la filosofía social en Alemania ha adoptado el papel de una disciplina subsidiaria. Esto refleja, de alguna forma cierta pérdida de su identidad; por ello carece de un ámbito objetual autónomo, y en su defecto se ha convertido en "una especie de vía lateral de la filosofía política”. En consecuencia, la filosofía social en la actualidad enfrenta una situación tempestuosa. Con el fin de evitar esta problemática, gracias a Rousseau fundador de la fi- losofía social moderna, es dable considerar la forma original de existencia del individuo, en contraste con su situación actual plena de patologías, que minimizan su libertad. Esto ha aportado un criterio de comparación frente al desarrollo humano y ha propiciado un espacio para una de las pocas alternativas de empiria, que le quedan a la filosofía social. Debe notarse que Rousseau y Hegel expresan su interés por la sana autorrealización del individuo, como una posibilidad de facilitar su reconocimiento como un ser digno en la sociedad. Así, para el primero esto se puede lograr mediante un estado de autarquía, en tanto que para el segundo, esta aspiración se enfoca en un bien común, que contribuya a formar una sociedad capaz de posibilitar a sus miembros la autorrealización, bajo las condiciones de los principios modernos de libertad.

En una fase posterior a lo que se acaba de anotar, Rousseau formaliza radicalmente la visión aristotélica, en cuanto que ya no se habla más de los fines de autorrealización humana dados por la naturaleza, sino solo de las condiciones que habilitan esta autorrealización; y por otra parte, ya no debe ser el Estado, sino la sociedad que se separa lentamente de éste, la que se interroga sobre las condiciones que entorpecen la realización de la vida humana. De esta manera emerge la filosofía social, como representante de una perspectiva ética en un nuevo terreno de la sociedad, que se empieza a constituir y que conlleva entre otras cosas, el estudio de las patologías sociales, que implica abordar lo relativo 
al conflicto, criterios para validar lo bueno, lo exitoso, lo ético, etc., temas bastante espinosos por cierto.

Por lo anterior, el futuro de la filosofía social depende de la posibilidad de justificar los juicios éticos atinentes a las condiciones necesarias de la vida humana, escenario que permite abordar lo referente a las potencialidades de la filosofía social hacia el futuro. Éstas son posibles a partir de los estudios de Honneth sobre el reconocimiento para garantizar la autorrealización del ser humano, independientemente de su adscripción social. Dicho reconocimiento no solo ha de tener una naturaleza distributiva de lo material, sino que también ha de incorporar un tratamiento adecuado, que permita superar el padecimiento, la humillación, la discriminación, etc. para garantizar la inclusión de los individuos a la sociedad, como seres dignos y con derechos a una vida buena. Para tal fin, Honneth procura establecer un ligamento fuerte y necesario entre el componente histórico y el antropológico; el primero siempre ha de recurrir a la extensa producción de la antropología para generar los marcos analíticos y metodológicos, que viabilicen los análisis de contexto y hagan más comprensibles sus relatos. 


\section{Referencias}

De Bono, E. (1986). El pensamiento lateral. Buenos Aires, Argentina: Vergara Granica.

Grueso, D. (2008). Tres modos de involucrar el reconocimiento en la justicia. Cali, Colombia: Universidad del Valle.

Grueso, D. (2015). La recuperación de Hegel por parte de Axel Honneth. En Reconocimiento y democracia desafíos de la justicia (p.p. 21-39). Cali, Colombia: Universidad del Valle.

Grueso, D. (2018). Apuntes de clase. Cali, Colombia: Universidad del Valle.

Honneth, A. (2011). La sociedad del desprecio. Madrid, España: Trota.

Londoño, C. (20015). Habermas entre la Teoría Crítica y el Liberalismo. En Reconocimiento y democracia desafíos de la justicia (p.p. 101-131). Cali, Colombia: Universidad del Valle.

Mèlich, J. C. (2001). La ausencia del testimonio. Barcelona, España: Anthropos Editorial Rubi.

Miguez, E. (2012). Antropología e historia. Memoria americana, (20-1). Recuperado de: http://www.scielo.org.ar/scielo.php?script=sci_arttext\&pi$\mathrm{d}=$ S1851-37512012000100009

Platón. (1966). Obras completas. (M. Araujo et al. Trads.). Madrid, España: Aguilar. Rousseau, J. (2005). Discurso sobre la desigualdad. Madrid, España: Editorial Tecnos. 